\title{
Deficits of brainstem and spinal cord functions after neonatal hypoxia-ischemia in mice
}

\author{
Blandine Bellot ${ }^{1,2}$, Julie Peyronnet-Roux', Catherine Gire ${ }^{2}$, Umberto Simeonii, ${ }^{2,3}$, Laurent Vinay ${ }^{1}$ and Jean-Charles Viemari ${ }^{1}$
}

\begin{abstract}
BACKGROUND: Perinatal cerebral hypoxia-ischemia (HI) can lead to severe neurodevelopmental disorders. Studies in humans and animal models mainly focused on cerebral outcomes, and little is known about the mechanisms that may affect the brainstem and the spinal cord. Dysfunctions of neuromodulatory systems, such as the serotonergic $(5-\mathrm{HT})$ projections, critical for the development of neural networks, have been postulated to underlie behavioral and motor deficits, as well as metabolic changes.
\end{abstract}

METHODS: The aim of this study was to investigate brainstem and spinal cord functions by means of plethysmography and sensorimotor tests in a neonatal Rice-Vanucci model of $\mathrm{HI}$ in mice. We also evaluated bioaminergic contents in central regions dedicated to the motor control of autonomic functions.

RESULTS: Mice with cerebral infarct expressed motor disturbances and had a lower body weight and a decreased respiratory frequency than SHAM, suggesting defects of brainstem neural network involved in the motor control of feeding, suckling, swallowing, and respiration. Moreover, our study revealed changes of monoamine and amino acid contents in the brainstem and the spinal cord of HI mice.

CONCLUSION: Our results suggest that monoaminergic neuromodulation plays an important role in the physiopathology of HI brain injury that may represent a good therapeutic target.

$\mathrm{t}$ is well documented that most cases of term neonatal encephalopathy are highly related to hypoxic-ischemic (HI) brain injury that occurs in utero or during delivery from a variety of intrapartum conditions (1). HI causes a combination of white and gray matter damage. Enlarged ventricules, loss of vulnerable oligodendrocyte progenitor cells, periventricular leucomalacia, astrogliosis, and microgliosis are typi$\mathrm{cal}$ features of $\mathrm{HI}$ damage $(1,2)$. The neurological outcome in surviving children is variable, ranging from mild cognitive impairment to major disabilities including cerebral palsy, behavioral disorders, and motor deficits (3). Early MRI with diffusion tensor imaging in neonates after $\mathrm{HI}$ is increasingly used to obtain clinical information noninvasively and follows the brain injury (4). It is well established that there are volumetric reductions in certain brain areas of $\mathrm{HI}$ preterm infants including the thalamus, basal ganglia, and cerebral cortex. Only a few studies have examined neuronal injury in the brainstem in human neonates (5) or in animal models (6). Until now, no investigation has focused on the possible spinal cord defects following HI.

Moreover, dysfunctions of some neuromodulators, such as the serotonergic system (5-HT) that is critical for the development of neuronal networks have been postulated to underlie behavioral and motor deficits (7). In addition, dysfunction of 5-HT neurotransmission is implicated in a host of physiological, metabolic, and behavioral changes in disease states such as epilepsy, Rett syndrome, Prader-Willi syndrome, and sudden infant death syndrome $(8,9)$.

The aim of this study was to investigate HI damage and the neurotransmitters system in the brainstem and the spinal cord in a model of neonatal HI in mice. We used the RiceVanucci model (10) of HI to investigate 1 and $2 \mathrm{wk}$ after the HI insult the neurological outcome evaluated by multiple approaches such as sensorimotor and behavioral tests (11). We also performed high-performance liquid chromatography (HPLC) measurements of monoamines and amino acids levels in the brainstem, in the pons and the medulla, and in the spinal cord. We extended our study to the juvenile mice (2 wk after the HI insult) to determine whether the early dysfunctions persist. We investigated respiration, an activity that is generated and modulated by nuclei within the brainstem (12), by means of plethysmographic recording 1 and $2 \mathrm{wk}$ after the insult.

Our results show that a $\mathrm{HI}$ insult on postnatal day 7 induces changes to the 5-HT, noradrenaline (NA), and dopamine (DA) contents in the brainstem and the spinal cord. $\gamma$ aminobutyric acid and glutamate contents are also affected in the brainstem. The sensorimotor assessment revealed early and long-lasting motor disturbances after HI insult. To conclude, our results suggest that neuromodulator disturbances occur after $\mathrm{HI}$ and may be involved in the motor deficits observed after $\mathrm{HI}$ brain injury. The neurotransmitter homeostasis needs to be finely 


\section{Articles $\mid$ Bellot et al.}

tuned after such insult and can represent a good therapeutic target to restore the function of different systems within the brainstem and the spinal cord after HI.

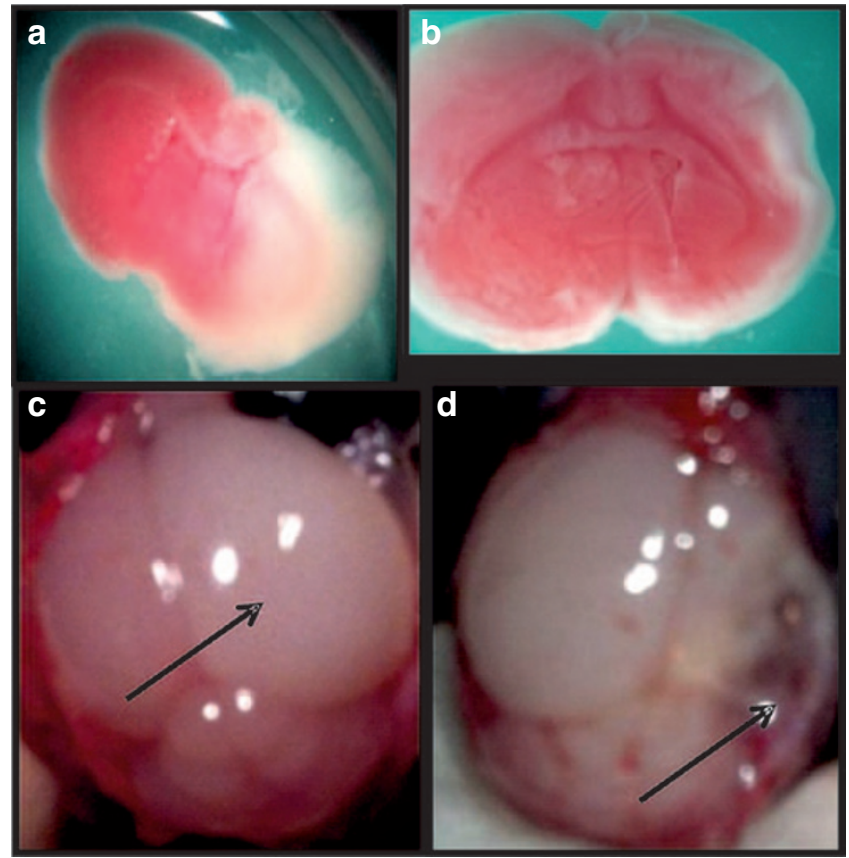

Figure 1. Evaluation of the brain infarct volume. Triphenyl-tetrazolium chloride-stained cortical slices from (a) a P8 mouse with cerebral infarct and from (b) a P8 SHAM. Macroscopic evaluation of the brain infarct (black arrows) (c) $1 \mathrm{~d}$ and (d) $1 \mathrm{wk}$ after hypoxia-ischemia.

\section{RESULTS}

Cerebral HI produced extensive cortical and subcortical cerebral infarcts ipsilateral to the cauterized carotid artery. After behavioral assessment, animals were sacrificed and their brain dissected out (Figure 1). The volume of the infarct being highly variable (13), we defined different populations: SHAM, animals with and without cerebral infarct respectively (Figure 1a,b). In this study, we focused on animals presenting massive ipsilateral hippocampal and cortical (Figure 1a) damages as revealed by triphenyl-tetrazolium chloride staining and previously reported by Stone et al. (14).

\section{Early Postural Deficits}

Short-term neurofunctional outcome has been previously described in details 1 and $24 \mathrm{~h}$ after the HI insult (13). Here, we confirmed that mice with cerebral infarcts (HI mice) have impaired righting and cliff aversion reflexes. We analyzed spontaneous locomotion by counting the number of steps after tail stimulation. Mice with cerebral infarcts $(n=20)$ had a significantly reduced mobility compared with mice without cerebral infarcts $(n=11)$ or SHAM $(n=22 ; 2.5 \pm 0.3$ vs. $4.8 \pm 1.3$ and $7.2 \pm 1.8$ steps, respectively, Tukey test, $P<0.05$ ). We then investigated the posture in mice with cerebral infarcts as described by Pflieger et al. (15). The angle between the foot axis (toe-heel) and the body axis (navel-anus line) was measured for the left and right feet ( $\alpha \mathrm{L}$ and $\alpha \mathrm{R}$, respectively, Figure 2a). These angles were used to estimate the "postural stability" (Figure 2b) and "postural asymmetry" (Figure 2c). We found

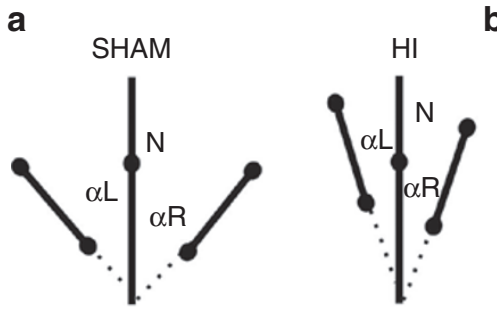

d

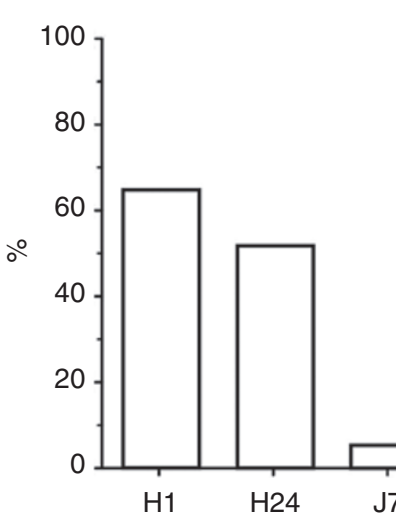

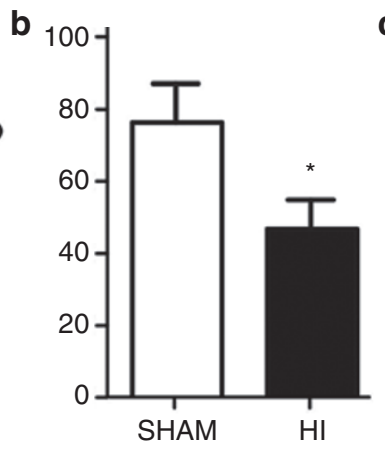

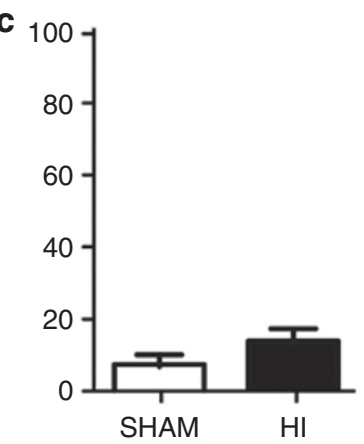

Fostural deficits in mice with cerebral infarcts. (a) Graph illustrating the positions of hindfeet relative to the nave in SHAM and mice with cerebral infarct. Angles between the body axis and the left ( $\alpha \mathrm{L})$ or the right ( $\alpha \mathrm{R})$ foot axis were measured. Histograms illustrating (b) the postural stability and (c) the postural asymmetry ( $t$-test, ${ }^{*} P<0.05$, hypoxia-ischemia $(\mathrm{HI}) n=12$, SHAM $\left.n=6\right)$. (d) Histogram illustrating the percentage of mice presenting a circling behavior in SHAM ( $n=25$, white bars) and in mice with $\left(n=21\right.$, black bars) and without $\left(n=17\right.$, gray bars) cerebral infarct $\left(\chi^{2}\right.$ test, $\left.* * P<.01\right)$. 
that the postural stability was reduced in mice with cerebral infarct $(n=12)$ compared with SHAM $(n=6 ; 47 \pm 8$ vs. $76 \pm 11$, $t$-test, $P<0.05)$, but postural asymmetry was similar.

We investigated the brainstem function such as respiration and feeding (as revealed by body weight increase). The respiratory frequency, measured by means of plethysmography, was significantly lower in mice with cerebral infarct $(n=61)$ compared with SHAM ( $n=39 ; 237 \pm 5$ vs. $259 \pm 7$ c/min, $t$-test, $P<0.01)$. The tidal volumes as well as the variability of the breathing cycle were not significantly affected at all ages.

\section{Long-Lasting Motor Deficits}

One and two weeks after the HI insult, mid- and long-term motor dysfunctions were investigated. Locomotor function was assessed using beam walking and vertical climbing tests. Mice with cerebral infarcts $(n=4)$ traveled a shorter distance on the beam compared with mice without infarct $(n=12)$ or SHAM $(n=12 ; 7.5 \pm 3.2 \mathrm{~cm}$ vs. $40.4 \pm 9.8 \mathrm{~cm}$ and $77.5 \pm 4.7 \mathrm{~cm}$, respectively, Tukey test, $P<0.001$, Figure $3 \mathrm{a}$ ). They also had an increased number of hind limb slips (Tukey test, no difference, Figure 3c). The ability of the mice with cerebral infarct to climb was also affected, that is, $67 \%$ of the mice with infarcts did not climb whereas all the SHAM climbed. Moreover, mice with cerebral infarcts that presented a postural abnormality also exhibited a cycling behavior $1 \mathrm{wk}$ after the infarct $\left(\chi^{2}\right.$ test, $P<0.01$, Figure 2d). At this stage, the respiratory frequency was lower in mice with cerebral infarct compared with SHAM (278 \pm vs. $302 \pm 9$ c/min, $t$-test, $P<0.05, n=43$ and $n=15$
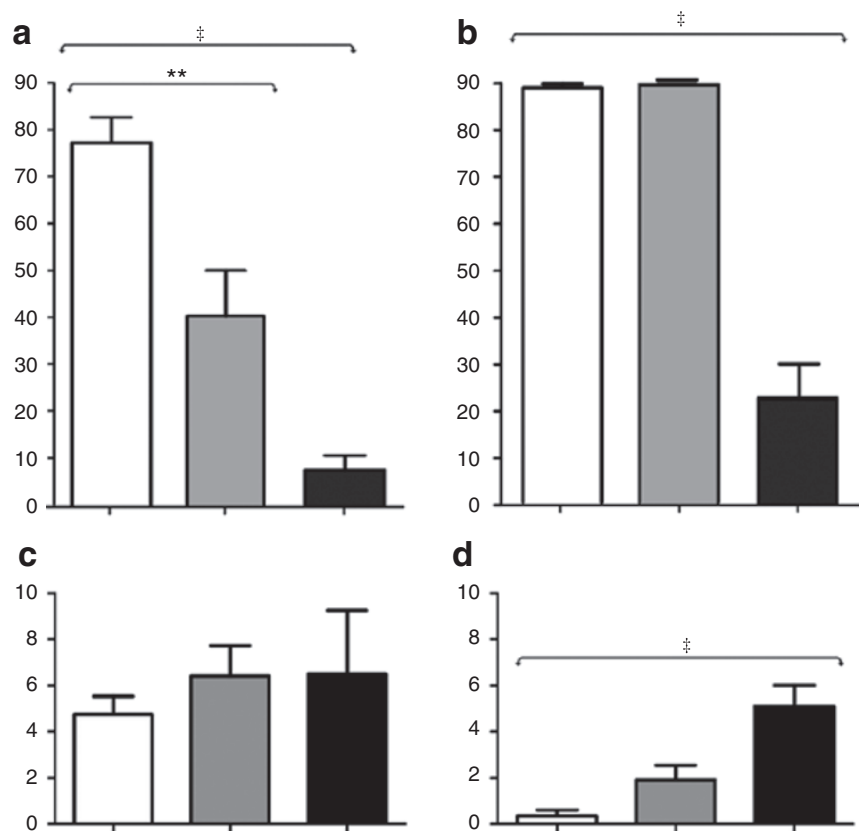

Figure 3. (a,b) Histograms illustrating the distance traveled on the beam in SHAM (respectively, D7: $n=12$, D14: $n=14$, white bars) and in mice with (respectively, $n=4$ and $n=12$, black bars) and without (respectively, $n=12$ and $n=11$, gray bars) cerebral infarct (a) 1 wk and (b) 2 wk after the insult (Tukey test, $\left.{ }^{\neq} P<0.001,{ }^{* *} P<0.01\right)$. (c,d) Histograms illustrating the number of hind limb slips on the beam in SHAM (white bars) and in mice with (black bars) and without (gray bars) cerebral infarct (c) $1 \mathrm{wk}$ and (d) 2 wk after the insult (Tukey test, $\neq P<0.001$ ). respectively, Figure 4a). We repeated the same test on juvenile mice (P21), and the motor deficits observed 1 wk after the HI insult persisted. Mice with cerebral infarct $(n=12)$ traveled a shorter distance on the beam compared with SHAM $(n=14$; $22.9 \pm 7.5$ vs. $89.3 \pm 0.7 \mathrm{~cm}$, Tukey test, $P<0.001$, Figure $3 b)$. The number of hind limb slips was significantly increased compared to SHAM $(5.1 \pm 0.9$ vs. $0.3 \pm 0.2, n=12$ and $n=14$ respectively, Tukey test, $P<0.001$, Figure $3 \mathrm{~d})$. However, the mice without infarct $(n=11)$ traveled the same distance as the SHAM. The ability of the mice with cerebral infarcts to climb remained affected, that is, $70 \%$ of the mice with infarcts did not climb $(n=12)$. The respiratory frequency remained also lower in mice with cerebral infarct $(n=10)$ compared with SHAM ( $n=4 ; 279 \pm 17$ vs. $315 \pm 20 \mathrm{c} / \mathrm{min}$, Figure 4a). As an assessment of feeding, suckling, and swallowing, we weighed the mice on the day of surgery as well as 1 and 2 wk later. The weight in the three groups increased significantly with age $(P<0.01$, nonparametric Spearman test). However, the slopes of the lines differed significantly, showing that the weight
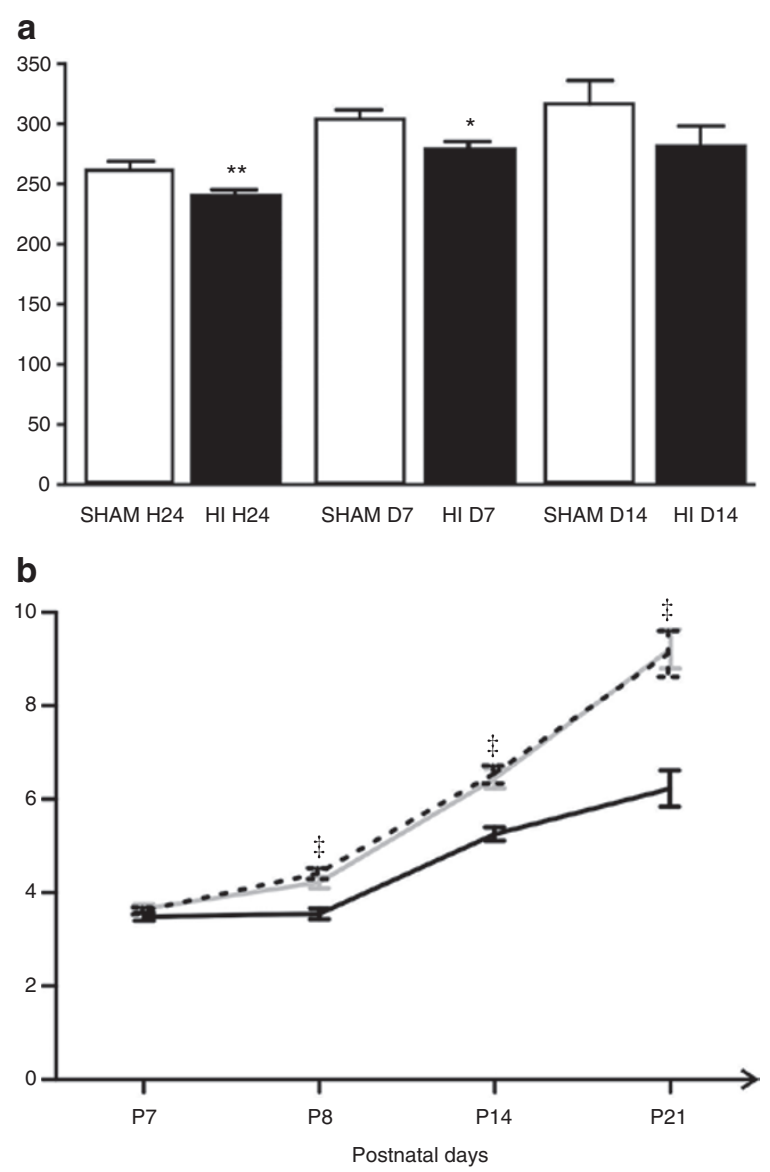

Figure 4. Decreased respiratory frequency in mice with cerebral infarcts. (a) The respiratory frequency is decreased in mice with cerebral infarct 1 d, 1 wk, and 2 wk after HI (respectively, SHAM: $n=39,15,4$ and HI: $n=61$, $43,10, t$-test, $\left.{ }^{* *} P<0.01,{ }^{*} P<0.05\right)$. (b) Graph illustrating the weight of the mice with cerebral infarct (solid line), without cerebral infarct (gray line), and SHAM (dotted line). The body weight of mice with cerebral infarct ( $n=31$ at P7, $n=31$ at P8, $n=36$ at P14, $n=19$ at P21) remained lower at all ages compared with SHAM ( $n=26$ at P7, $n=26$ at P8, $n=35$ at P14, $n=15$ at P21) (Tukey test, $\left.{ }^{\ddagger} P<0.001\right)$. 
increase was significantly larger in SHAM. The difference between the HI with cerebral infarct $(n=26)$ and the SHAM group $(n=31)$ became significant on the day after the brain injury, and the weight of $\mathrm{HI}$ animals remained lower at all ages (at P8, $3.51 \pm 0.11$ vs. $4.41 \pm 0.11 \mathrm{~g}, P<0.001$; at $\mathrm{P} 14,5.22 \pm 0.14$ vs. $6.53 \pm 0.19 \mathrm{~g}, P<0.001$; and at $\mathrm{P} 21,6.20 \pm 0.41$ vs. $9.1 \pm 1.0 \mathrm{~g}$, $P<0.001$, Tukey test, Figure 4b). There was no significant difference between mice without infarct and SHAM.

\section{Bioaminergic Disturbances in the Brainstem and the Spinal Cord of HI Mice}

DA, NA, and 5-HT contents were evaluated by HPLC in the pons, medulla, and spinal cord of mice with cerebral infarct $(n$ $=7)$ and SHAM $(n=9) .5$-HT, NA, and DA contents were not affected in the pons of mice with cerebral infarct. 5-HT contents were increased in both the medulla (170 \pm 7 vs. $138 \pm 7$ $\mu \mathrm{mol} / \mathrm{l}$, Mann-Whitney test, $P<0.05)$ and the spinal cord (50 \pm 4 vs. $26 \pm 3 \mu \mathrm{mol} / \mathrm{l}$, Mann-Whitney test, $P<0.001$ ) of mice with cerebral infarcts (Figure $5 \mathbf{a}, \mathbf{b}$ ). DA and NA contents were also affected but only in the spinal cord: NA contents were significantly increased ( $65 \pm 3$ vs. $45 \pm 4 \mu \mathrm{mol} / \mathrm{l}$, MannWhitney test, $P<0.01$, Figure $5 \mathrm{~b}$ ), whereas DA contents were significantly decreased ( $9 \pm 1$ vs. $6 \pm 2 \mu \mathrm{mol} / \mathrm{l}$, Mann-Whitney test, $P<0.05$, Figure 5b).

We assessed the turnover of 5 -HT by calculating the ratio 5-HIAA/5-HT. The ratio 5-HIAA/5-HT was significantly decreased in all structures examined $(2 \pm 0.1$ vs. $2.6 \pm 0.2$,
Mann-Whitney test, $P<0.05$ in the pons; $1.54 \pm 0.08$ vs. $2.22 \pm 0.11$, Mann-Whitney test, $P<0.001$ in the medulla and $1 \pm 0.1$ vs. $2.4 \pm 0.2$, Mann-Whitney test, $P<0.001$ in the spinal cord, Figure 5c).

We then investigated the $\gamma$ aminobutyric acid, glutamate, and aspartate contents in the same structures. The levels of all three amino acids were decreased in the medulla of mice with cerebral infarct $(45 \pm 2$ vs. $36 \pm 3 \mu \mathrm{mol} / \mathrm{l}$ respectively, Mann-Whitney test, $P=0.05 ; 1151 \pm 68$ vs. $770 \pm 67 \mu \mathrm{mol} / \mathrm{l}$, Mann-Whitney test, $P<0.01 ; 151 \pm 5$ vs. $111 \pm 10 \mu \mathrm{mol} / \mathrm{l}$, Mann-Whitney test, $P<0.01$, Figure 6).

\section{DISCUSSION}

Neonatal cerebral HI is an important cause of brain injury that leads to high rates of mortality or long-term neurological morbidity. HI injury in 7-d-old mice induces cognitive deficits and impairs motor performance (16). Here, we confirmed that sensorimotor reflexes are affected $24 \mathrm{~h}$ after $\mathrm{HI}$ insult as previously described (13). We extended our study of motor functions 1 and 2 wk after the insult. Our results revealed that $1 \mathrm{wk}$ after the cerebral infarct, mice had a lower performance in the beam-walking test (shorter traveled distance and higher number of hindlimb slips). We showed that feeding behavior as well as respiration is also affected. Finally, we correlated these motor defects with HPLC measurements in the brainstem and in the spinal cord $1 \mathrm{wk}$ after the infarct. Our results showed that monoamine as well as amino acid contents were

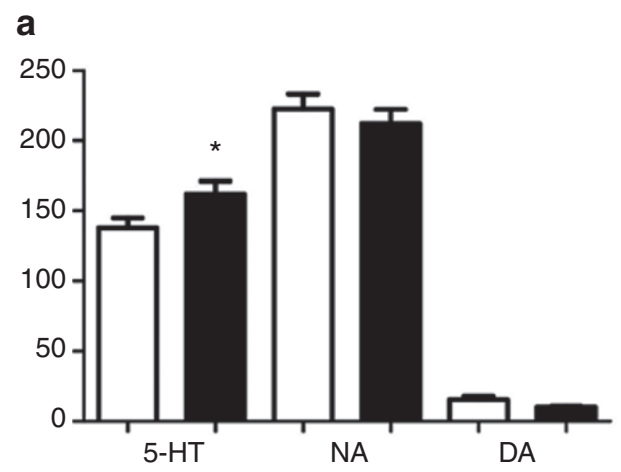

b

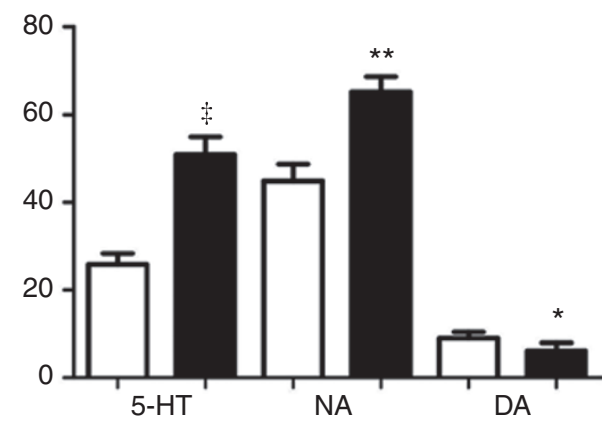

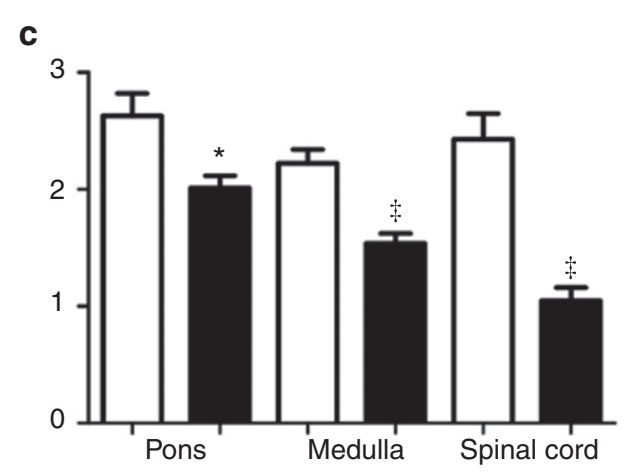

Figure 5. Disturbances in monoamine content in mice with cerebral infarcts. (a) Histogram illustrating the level of 5-HT, noradrenaline (NA), and dopamine (DA) in the medulla of SHAM (white fill, $n=9$ ) and of HI mice with cerebral infarcts (black fill, $n=7)\left(\right.$ Mann-Whitney test, $\left.{ }^{*} P<0.05\right)$. (b) Histogram illustrating the level of 5-HT, NA, and DA in spinal cord of SHAM $(n=9)$ and of hypoxia-ischemia (HI) mice with cerebral infarcts $(n=7)($ Mann-Whitney test, $\left.{ }^{\ddagger} P<0.001,{ }^{* *} P<0.01,{ }^{*} P<0.05\right)$. (c) Histogram illustrating the 5 -HT turnover $(5-\mathrm{HIAA} / 5-\mathrm{HT})$ in mice with cerebral infarcts $(n=7)$ and in SHAM $(n=9)$, in the pons, the medulla, and the spinal cord (Mann-Whitney test, ${ }^{\ddagger} P<0.001,{ }^{*} P<0.05$ ). 
a

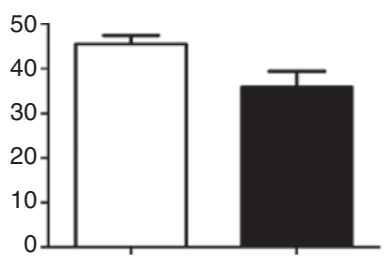

b

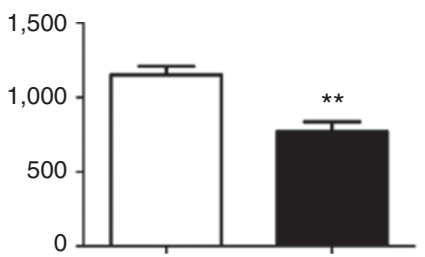

C

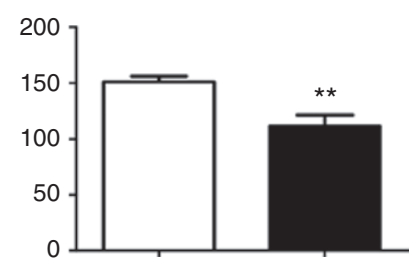

Figure 6. Disturbances in amino acid content in mice with cerebral infarcts. Histograms illustrating the level of (a) $\gamma$ aminobutyric acid, (b) glutamate, (c) and aspartate in the medulla of SHAM (white fill, $n=9$ ) and of hypoxia-ischemia (HI) mice with cerebral infarcts (black fill, $n=7)\left(\right.$ Mann-Whitney test, ${ }^{* *} P$ $<0.01)$.

disrupted. Taken together, we propose that disruption of the 5-HT system in the brainstem and the spinal cord may account for the motor defects observed in HI mice.

\section{Possible Brainstem Dysfunctions}

Feeding and respiration are vital functions for newborns and children. These complex motor functions originate from central pattern generator localized in the brainstem $(8,12)$ which is vulnerable to HI (17). The respiratory center is located in an area within the brainstem called the pre-Bötzinger complex which is continuously modulated by endogenous bionamines (8). Here, we investigated the respiratory function in $\mathrm{HI}$ mice $1 \mathrm{wk}$ after the brain infarct. Plethysmographic recordings revealed a depressed respiratory frequency suggesting a dysfunction of the respiratory network and its bioaminergic modulation since 5-HT contents were modified in the medulla. However, we do not know whether neurons of the central pattern generator for respiration are affected by HI. Brainstem involvement in hypoxic or hypotensive brain damage is acknowledged, and infants with birth asphyxia have respiratory failure and are more vulnerable to sudden death syndrome (18). Then, we weighed the mice to revealed feeding problems. HI mice had a lower body weight compared with SHAM, which suggests that the motor control of feeding, suckling, or swallowing within the brainstem (19) might be affected which is in agreement with observations made on preterms or neonates who suffered from HI encephalopathy (17). The brainstem is a typical site of injury, and brainstem injury is considered a key predictor of death in term and preterm infants with severe HI encephalopathy (4). Sixty-five percent of children who survive after the neonatal period have feeding impairment (20). Altogether, feeding as well as respiratory impairments are linked to the presence and the severity of injury revealed with MRI (20). In our study, we did not reveal macroscopic lesions of the brainstem which has however been reported in children with $\mathrm{HI}$ (21). Our HPLC measurements clearly showed that the monoaminergic and amino acids systems within the brainstem and the spinal cord are affected. It is important to note that brainstem injury is presumably secondary to primary injury events in the forebrain because the brainstem is outside the vascular field of the carotid artery and would not be affected by immediate changes in perfusion after carotid coagulation or ligation (22). Two phases can be defined after neonatal HI insult: first, an early phase within $24-48 \mathrm{~h}$, during which excitotoxic damages causing calcium overload occur, leading to cell death.
High levels of free radicals also cause irreversible injury. Then, a second phase that can last for weeks or months takes place. During this phase, increased number of activated microglia, astrogliosis, and increased levels of proinflammatory cytokines have been described and identified as features of neuroinflammation (for review, see Buller et al. (6)). Furthermore, inhibition of neuroinflammation in the forebrain but not in the brainstem reduces the HI-induced raphé 5-HT losses (23). 5-HT raphé fibers projecting to the forebrain but not fibers that project to the medulla and the spinal cord (fibers from the raphé magnus, palidus, and obscurus) are disrupted after HI $(6,24)$. We then proposed that the release of 5 -HT in the medulla and the spinal cord might be affected since the 5-HT contents were modified in these regions after HI. Since a local neuroinflammatory response in the brainstem does not seem to account for the observed changes (23), it is conceivable that other mechanisms dependent on neural connections or neural excitability may account for remote secondary injury in the brainstem. Yet, raphé neurons not directly affected by HI could express differential activity due to changes in their close environment. Thus, the mechanisms that might be responsible for these changes need to be investigated.

\section{Disturbances of the 5-HT, NA, and DA System in HI Mice}

The monoaminergic systems such as serotonergic innervations, develop very early during gestation and is one of the first neurotransmitter to appear in the developing brain (25). Its trophic action is particularly important for the maturation of neuronal networks such as the locomotor circuitry, the suction-swallowing pattern, and the respiratory network (26). The vulnerability of raphé serotonergic neurons to HI depends on their rostrocaudal distribution. It has been recently reported that 5-HT cortex-projecting neurons were decreased in the dorsal, ventrolateral, and ventral raphe nuclei after HI at P3 (6). Other raphé nuclei may survive because they project to other brain regions that are not directly affected by HI. However, in this study, we showed that 5-HT, NA, and DA contents are significantly affected in the brainstem and the spinal cord in mice with cerebral infarcts. We performed HPLC measurements in mice with similar infarct injury to minimize variability of the neurological outcome (13). Moreover, the 5-HT turnover is significantly affected in the pons, the medulla, and spinal cord of HI mice. In other studies, where the 5HIAA concentrations in the cerebrospinal fluid (27) and monoamine oxidase activity in platelets (27) were abnormal such as the Prader-Willi 
syndrome respiratory defects have been observed (28). Taken together, perinatal depletion or excess of 5-HT $(8,15)$ results in dysfunctions of neural networks. The 5-HT system is not the only one vulnerable to HI and specific loss of catecholaminergic neurons has been previously described following $\mathrm{HI}$ at P3, in the ventrolateral medulla and the nucleus tractus solitaries (29). In this study, we also showed significant changes in NA content in the medulla and in the spinal cord as well as for the DA content. This shows the important contribution of monoamines in the maturation and operation of neural networks and suggests that monoamines could also been involved in the physiopathology of HI. Finally, we propose that disruption of the 5-HT, NA, and DA systems may underlie impairments such as cardiorespiratory, cognitive, and motor deficits, observed $1 \mathrm{wk}$ after the HI insult. In addition, these deficits may also be responsible for motor defects observed in children who have experienced neonatal HI (9).

\section{Other Disturbances}

GABAergic and glutamatergic neurons are important for feeding behavior (30) and respiration. The respiratory rhythm is generated by the interaction of two coupled hindbrain oscillators considered to be responsible for respiratory rhythm that contains glutamatergic neurons. Moreover, depressed respiratory frequency and irregular breathing observed in knockout mice for the gene chx10 likely result from insufficient glutamatergic drive to the medullary respiratory network (31). Concerning feeding, radiofrequency lesions of nondopaminergic neurons of the midbrain produce marked aphagia (32). In our study, glutamate and aspartate in the brainstem are affected by HI, which may cause respiratory and feeding problems.

\section{Animal Model and Clinical Relevance}

The model of neonatal $\mathrm{HI}$ as described by Rice et al. (10). has been extensively used to investigate cognitive and sensorimotor functions; however, it should be emphasized that depending on the age of the insult, the results should be cautiously discussed. Previous studies interested in the 5-HT systems have used the rat model at P3 (6). Although still debated, P7 as corresponds to a brain development of 3436 wk gestational age in the human fetus $(10,33)$. P3 corresponds to a quite immature brain, and the effects of HI may be different than those observed at P7. The size of the lesion might also be considered since in infants diffuse lesions have been described $(21,34)$, whereas in this model, the brain injury affects the ipsilateral hemisphere. Finally, this model induces a wide range of disturbances that resulted from $\mathrm{HI}$ lesions including the sensorimotor cortex, but whether this model reflects cerebral palsy can be extensively discussed. Lesions of the cortex may also account for respiratory and motor disturbances observed in HI lesions, but it is difficult to interpret the role of the cortex in the generation of breathing which is acknowledged to be devoted to the brainstem. However, it is very well known that common brainstem areas such as the rostral, dorsal, and inferior ventral pons integrate respiratory control mediated by conscious or unconscious mechanism $(35,36)$. This model allows the investigation of possible mechanisms following cerebral injury during the neonatal period, which is critical for the understanding of neonatal HI. Here, we showed for the first time that neurotransmitter systems in the brainstem and in the spinal cord are affected and represent potential targets for future therapeutic treatments.

\section{METHODS}

C57/BL6J mice pups of both genders were used in this study. All research was done according to a protocol approved by the Institut de Neurosciences de la Timone ethical committee (authorized number 71) and in accordance with national charter concerning the animal experiment's ethics.

Murine Model of HI encephalopathy, the "Rice-Vannucci Model" On postnatal day 7 (P7), pups were assigned randomly to SHAM or HI groups. Mice were anesthetized using $2 \%$ isoflurane inhalation. Surgical procedure was made at room temperature $\left(25^{\circ} \mathrm{C}\right)$ under binocular microscopy within $5 \mathrm{~min}$. The right common carotid artery was isolated and cauterized by bipolar heating forceps. The mice were allowed to recover for $3 \mathrm{~h}$. Mice were then exposed to $8 \% \mathrm{O} 2$ for $45 \mathrm{~min}$ at $37-38{ }^{\circ} \mathrm{C}$ (temperature was measured with a thermometer placed in the chamber) in a tightly closed humidified chamber (controlled by Pro-OX sensor). Following hypoxia, pups recovered for $1 \mathrm{~h}$ in the same chamber maintained at $37-38^{\circ} \mathrm{C}$ breathing room air. SHAM group animals underwent only surgery without carotid artery cauterization and then hypoxia for $45 \mathrm{~min}$ like the HI group.

\section{Anatomic Assessment of Cerebral Injury}

To evaluate the brain infarct, mice brains were sectioned into coronal slices and immersed in triphenyl-tetrazolium chloride (Sigma Aldrich, Saint-Quentin, France) (13). Triphenyl-tetrazolium chloride is taken up into living mitochondria, which converts it to a red color. So, after incubation in $37^{\circ} \mathrm{C}$ during $30 \mathrm{~min}$, viable tissue stains brick red, and infarcted tissue can be identified by the absence of staining (Figure 1a,b). One and two weeks after the HI insult, we identified the lesion by macroscopic observation. We then defined two subgroups among the HI group: (i) HI with cerebral infarct in which a lesion could be observed (Figure 1c,d) and (ii) HI without cerebral infarct which had no macroscopic lesion.

\section{Short-Term Outcome Evaluation}

Twenty-four hours after the insult, we counted the number of steps after tail stimulation. We also studied the pup's posture. The postural stability was estimated by the difference in absolute value from the right angle $(\alpha \mathrm{R})$ and left angle $(\alpha \mathrm{L})$ representing the angle between the foot axis and body axis (line between the navel, the anus, and the face (Figure 2a)). The postural asymmetry was estimated by adding $\alpha \mathrm{R}$ and $\alpha \mathrm{L}$ angles (absolute values). Finally, we considered the circling behavior.

\section{Mid-Term Outcome Evaluation}

To estimate HI mid-term outcome, two behavioral tests were done 7 days (D7) and 14 days (D14) after HI insult.

Beam walking. This test was used to assess motor function estimating hind limbs coordination and balance. The beam-walking apparatus consisted of a wooden circular beam of $90 \mathrm{~cm}$, elevated $50 \mathrm{~cm}$ above the floor. Mice were allowed to walk on platforms located at each end of the beam. We calculated the distance (in centimeter) traveled by each mouse on the beam, and we counted the number of hindlimb slips.

Vertical climbing. This test investigated motor skill and labyrinthine functions. It evaluates the ability of mice to climb on a vertical grid. The score " 2 " is attributed if the animal crosses all the stitches successfully, " 1 " if it crosses some or if it moves in a side way, and " 0 " if it crosses none. Every mouse had three trials, and only the best score was kept for analysis. 


\section{Body Growth}

The pups weight was collected on the day of HI (P7), 24h, $7 \mathrm{~d}$ and 14 $\mathrm{d}$ later. We calculated the mean weight for each group.

\section{Plethysmography}

Ventilation was measured in awake and unrestrained HI and SHAM mice at H24, D7, and D14 using barometric plethysmography, according to the protocol described previously (37). The volume of the Plexiglas chambers was $0.04 \mathrm{l}$, and calibration of the chamber was obtained before placing the animal in the box in order to avoid signal interferences between calibration and ventilation of the animal. Respiratory frequency (f) was calculated from breath-by-breath records over 30-50 consecutive cycles (around $30 \mathrm{~s}$ ) by computer analysis of the spirogram. We made sure that all recordings were realized at a temperature $\left(33^{\circ} \mathrm{C}\right)$ corresponding to thermoneutrality. If necessary, the air was heated or cooled down before its entry in the plethysmograph. The set point of ambient temperature (thermoneutrality) was strictly the same for HI and for SHAM mice. The respiratory frequency is expressed in $\mathrm{min}^{-1}$.

\section{Tissue Preparation}

Mice cortex, brainstem, and spinal cord were taken on D7. After decapitation, lumbar spinal cord was obtained by extrusion in an artificial cerebrospinal fluid (containing (in mmol): $128 \mathrm{NaCl}, 4 \mathrm{KCl}, 1.5$ $\mathrm{CaCl}$, 1 MgSO 4, 0.5 NaH2PO4, 21 NaHCO3 and 30 glucose (Sigma Aldrich) oxygenated with 95\% O2/5\% CO2, pH 7.4). Lumbar spinal cord (T8-S4) was dissected out accordingly to previous description, frozen and then kept in $-80{ }^{\circ} \mathrm{C}(38)$. For the brainstem dissection, the pons and the medulla were separated as previously described (39). The pons includes A5 and A6 noradrenergic nuclei, whereas the medulla includes A1 and A2 noradrenergic nuclei as well as 5-HT neurons of the raphé magnus, palidus, and obscurus, which project to the brainstem and the spinal cord.

\section{Biochemistry}

5-HT, NA, and DA levels were quantified as well as $\gamma$ aminobutyric acid, glutamate, and aspartate level at brainstem and spinal level. The group with cerebral infarct was compared with SHAM. Dosages were done by Neurochem laboratory (Lyon, France) by HPLC technique (40), from brainstem samples (pons and medulla), and spinal cord samples frozen at $-80{ }^{\circ} \mathrm{C}$.

\section{Statistical Analysis}

Data are expressed as mean \pm SEM. The statistical tests GraphPad Prism 4 software (La Jolla, CA) used are given in the text and figure legends. For all tests, statistical significance was taken at $P<0.05$.

\section{ACKNOWLEDGMENTS}

We would like to thank Dr. Sandrine Parrot and Dr. Luc Denoroy from the NeuroChem facility (Université Claude Bernard, Lyon, France) for the highperformance liquid chromatography analysis and Dr. Pierre Roubertoux for the advice provided for the sensorimotor tests.

\section{STATEMENT OF FINANCIAL SUPPORT}

B.B. was supported by the Assistance Publique Hôpitaux de Marseille and Aix Marseille Université.

Disclosure: The authors declare no conflict of interest.

\section{REFERENCES}

1. Volpe JJ. Brain injury in premature infants: a complex amalgam of destructive and developmental disturbances. Lancet Neurol 2009;8:110-24.

2. Leviton A, Gressens P. Neuronal damage accompanies perinatal whitematter damage. Trends Neurosci 2007;30:473-8.

3. Shankaran S, Pappas A, McDonald SA, et al.; Eunice Kennedy Shriver NICHD Neonatal Research Network. Childhood outcomes after hypothermia for neonatal encephalopathy. N Engl J Med 2012;366:2085-92.

4. Martinez-Biarge M, Diez-Sebastian J, Kapellou O, et al. Predicting motor outcome and death in term hypoxic-ischemic encephalopathy. Neurology 2011;76:2055-61.

5. Logitharajah P, Rutherford MA, Cowan FM. Hypoxic-ischemic encephalopathy in preterm infants: antecedent factors, brain imaging, and outcome. Pediatr Res 2009;66:222-9.
6. Reinebrant HE, Wixey JA, Buller KM. Disruption of raphé serotonergic neural projections to the cortex: a potential pathway contributing to remote loss of brainstem neurons following neonatal hypoxic-ischemic brain injury. Eur J Neurosci 2012;36:3483-91.

7. Herlenius E, Lagercrantz H. Development of neurotransmitter systems during critical periods. Exp Neurol 2004;190:Suppl 1:S8-21.

8. Viemari JC, Tryba AK. Bioaminergic neuromodulation of respiratory rhythm in vitro. Respir Physiol Neurobiol 2009;168:69-75.

9. Kinney HC, Broadbelt KG, Haynes RL, Rognum IJ, Paterson DS. The serotonergic anatomy of the developing human medulla oblongata: implications for pediatric disorders of homeostasis. J Chem Neuroanat 2011;41:182-99.

10. Rice JE 3rd, Vannucci RC, Brierley JB. The influence of immaturity on hypoxic-ischemic brain damage in the rat. Ann Neurol 1981;9:131-41.

11. Sluyter F, Cohen-Salmon C, Carlier M, Cherif C, Verray FM, Roubertoux PL. Mitochondrial DNA and behavior: Implication of mitochondrial DNA in learning and memory. Behav Genet 2003;33:719-720.

12. Viemari JC. Noradrenergic modulation of the respiratory neural network. Respir Physiol Neurobiol 2008;164:123-30.

13. Ten VS, Bradley-Moore M, Gingrich JA, Stark RI, Pinsky DJ. Brain injury and neurofunctional deficit in neonatal mice with hypoxic-ischemic encephalopathy. Behav Brain Res 2003;145:209-19.

14. Stone BS, Zhang J, Mack DW, Mori S, Martin LJ, Northington FJ. Delayed neural network degeneration after neonatal hypoxia-ischemia. Ann Neurol 2008;64:535-46.

15. Pflieger JF, Clarac F, Vinay L. Postural modifications and neuronal excitability changes induced by a short-term serotonin depletion during neonatal development in the rat. J Neurosci 2002;22:5108-17.

16. McAuliffe JJ, Miles L, Vorhees CV. Adult neurological function following neonatal hypoxia-ischemia in a mouse model of the term neonate: water maze performance is dependent on separable cognitive and motor components. Brain Res 2006;1118:208-21.

17. Quattrocchi CC, Longo D, Delfino LN, et al. Dorsal brain stem syndrome: MR imaging location of brain stem tegmental lesions in neonates with oral motor dysfunction. AJNR Am J Neuroradiol 2010;31:1438-42.

18. Saito $\mathrm{Y}$, Ito M, Ozawa $\mathrm{Y}$, et al. Changes of neurotransmitters in the brainstem of patients with respiratory-pattern disorders during childhood. Neuropediatrics 1999;30:133-40.

19. Jean A. Brain stem control of swallowing: neuronal network and cellular mechanisms. Physiol Rev 2001;81:929-69.

20. Martinez-Biarge M, Diez-Sebastian J, Wusthoff CJ, et al. Feeding and communication impairments in infants with central grey matter lesions following perinatal hypoxic-ischaemic injury. Eur J Paediatr Neurol 2012;16:688-96.

21. Okereafor A, Allsop J, Counsell SJ, et al. Patterns of brain injury in neonates exposed to perinatal sentinel events. Pediatrics 2008;121:906-14.

22. Vannucci RC, Lyons DT, Vasta F. Regional cerebral blood flow during hypoxia-ischemia in immature rats. Stroke 1988;19:245-50.

23. Wixey JA, Reinebrant HE, Spencer SJ, Buller KM. Efficacy of post-insult minocycline administration to alter long-term hypoxia-ischemia-induced damage to the serotonergic system in the immature rat brain. Neuroscience 2011;182:184-92.

24. Reinebrant HE, Wixey JA, Buller KM. Neonatal hypoxia-ischaemia disrupts descending neural inputs to dorsal raphe nuclei. Neuroscience 2013; 248C:427-35.

25. Vinay L, Brocard F, Pflieger JF, Simeoni-Alias J, Clarac F. Perinatal development of lumbar motoneurons and their inputs in the rat. Brain Res Bull 2000;53:635-47.

26. Hodges MR, Wehner M, Aungst J, Smith JC, Richerson GB. Transgenic mice lacking serotonin neurons have severe apnea and high mortality during development. J Neurosci 2009;29:10341-9.

27. Akefeldt A, Ekman R, Gillberg C, Månsson JE. Cerebrospinal fluid monoamines in Prader-Willi syndrome. Biol Psychiatry 1998;44:1321-8.

28. Zanella S, Watrin F, Mebarek S, et al. Necdin plays a role in the serotonergic modulation of the mouse respiratory network: implication for Prader-Willi syndrome. J Neurosci 2008;28:1745-55. 


\section{Articles $\mid$ Bellot et al.}

29. Buller KM, Wixey JA, Pathipati P, et al. Selective losses of brainstem catecholamine neurons after hypoxia-ischemia in the immature rat pup. Pediatr Res 2008;63:364-9.

30. Narayanan NS, Guarnieri DJ, DiLeone RJ. Metabolic hormones, dopamine circuits, and feeding. Front Neuroendocrinol 2010;31:104-12.

31. Crone SA, Viemari JC, Droho S, Mrejeru A, Ramirez JM, Sharma K. Irregular Breathing in Mice following Genetic Ablation of V2a Neurons. J Neurosci 2012;32:7895-906.

32. Nadaud D, Simon H, Herman JP, Le Moal M. Contributions of the mesencephalic dopaminergic system and the trigeminal sensory pathway to the ventral tegmental aphagia syndrome in rats. Physiol Behav 1984;33:879-87.

33. Romijn HJ, Hofman MA, Gramsbergen A. At what age is the developing cerebral cortex of the rat comparable to that of the full-term newborn human baby? Early Hum Dev 1991;26:61-7.

34. Ashwal S, Tone B, Tian HR, Chong S, Obenaus A. Comparison of two neonatal ischemic injury models using magnetic resonance imaging. Pediatr Res 2007;61:9-14.
35. McKay LC, Adams L, Frackowiak RS, Corfield DR. A bilateral corticobulbar network associated with breath holding in humans, determined by functional magnetic resonance imaging. Neuroimage 2008;40:1824-32.

36. Pattinson KT, Mitsis GD, Harvey AK, et al. Determination of the human brainstem respiratory control network and its cortical connections in vivo using functional and structural imaging. Neuroimage 2009;44:295-305.

37. Peyronnet J, Roux JC, Geloën A, et al. Prenatal hypoxia impairs the postnatal development of neural and functional chemoafferent pathway in rat. J Physiol 2000;524 Pt 2:525-37.

38. Stil A, Jean-Xavier C, Liabeuf S, et al. Contribution of the potassium-chloride co-transporter KCC2 to the modulation of lumbar spinal networks in mice. Eur J Neurosci 2011;33:1212-22.

39. Viemari JC, Bévengut M, Coulon P, Hilaire G. Nasal trigeminal inputs release the A5 inhibition received by the respiratory rhythm generator of the mouse neonate. J Neurophysiol 2004;91:746-58.

40. Panayotis N, Ghata A, Villard L, Roux JC. Biogenic amines and their metabolites are differentially affected in the Mecp2-deficient mouse brain. BMC Neurosci 2011;12:47. 